\title{
Enhanced stimulus generalization of a food reinforced response to a CS for water ${ }^{1}$
}

\author{
THOMAS HYDE AND MILTON A. TRAPOLD \\ UNIVERSITY OF MINNESOTA
}

It was previously shown that the amount of stimulus generalization of a food reinforced instrumental response from a training stimulus to a generalization test stimulus is markedly increased if the test stimulus has been independently paired classically with food, and decreased if the test stimulus has been paired with active nonreinforcement. The present experiment showed similar transfer from classical to instrumental conditioning when a different reinforcer was used in classical conditioning than in instrumental conditioning.

A number of recent studies have shown that manipulations involving the simple pairing of stimuli with a known reinforcer (or absence thereof) can exert a strong influence upon the subsequent performance of instrumental responses that are under the control of these same stimuli and reinforcer. Such transfer of training from the classical to the instrumental learning paradigm has been shown in both the rate of free operant discrimination learning (Trapold, 1966; Bower \& Grusec, 1964) and in various aspects of performance on a discrete trial learning task (Trapold et al, 1967; Trapold \& Winokur, 1967).

While these studies have shown that reinforcement experiences encountered independently of a specific instrumental response can transfer to and affect the performance of that response, there has been little work directly concerned with the necessary and/or sufficient conditions for such transfer. This study is addressed to one aspect of this question, namely, whether it is necessary that the same reinforcer be employed in both classical conditioning and instrumental learning. All previous studies except Bower \& Kaufmann (1963) have used the same reinforcer in both training situations and, hence, the question arises whether classical $\rightarrow$ instrumental transfer depends upon this commonality.

One of the results reported by Trapold \& Winokur (1967) was that stimulus generalization of a well learned, food reinforced response from a training stimulus, $S$, to a generalization stimulus, S', was greater when $S^{\prime}$ has been previously paired classically with food than when not. The present experiment asked whether a similar enhancement of stimulus generalization would be found to a stimulus that had previously been paired classically with water reinforcement.

\section{Method}

Ss were eight naive male albino rats, approximately 120 days old at the beginning of the experiment. The apparatus consisted of an automatically programmed operant conditioning chamber equipped with a response lever, food and water delivery devices, bar light and house light, speaker, sound deadening enclosure, and control and recording equipment located in another room. Ss were first reduced to $80 \%$ of their ad lib weights by controlled-amount feeding once a day immediately following each daily session. Water was not available except for a $1 \mathrm{~h}$ period that began when $\mathrm{S}$ was given his daily food allotment. This procedure insured a high degree of both food and water deprivation. After two 1-h adaptation sessions in the experimental chamber, all Ss received five daily session of magazine training, in each of which $S$ received 25 food (.045 g) and 25 water $(.08 \mathrm{ml})$ reinforcements delivered noncontingently and irregularly intermixed on a variable interval schedule averaging $1 \mathrm{~min}(\mathrm{VI}-1)$. Water reinforcements were preceded by a .3 sec blackout of the bar light and food reinforcements by the normal operating sound of the feeder. All Ss were the given two sessions during which each press of the response bar was reinforced with a single food pellet to a total of 100 reinforcements per session. This was followed by 14 sessions of preconditioning with the bar removed from the chamber. Each session involved 24 3-sec presentations of each of two auditory stimuli (a 2000 cps tone and a 20 pps clicker) irregularly intermixed on a VI-1' schedule. One stimulus ( $\mathrm{S}+$ ) was consistently followed by water reinforcement and the other (S-) was not. For four $S s$ the tone served as $S_{+}$, for the other four, the clicker. The bar was then reinserted into the chamber and all Ss received five 48-trial sessions of training on a discrete trial bar pressing task for food reinforcement. A trial began with the onset of the house light. A single bar press terminated the house light and delivered a single pellet. Response latency was recorded on each trial. The intertrial interval was variable with a nominal average of $1 \mathrm{~min}$. However, to this was added the condition that no trial would begin until at least $20 \mathrm{sec}$ had passed without an intertrial response. This procedure rapidly eliminates intertrial responses and such responses will hereafter not be considered. Following discrete trial bar press training, all Ss received five sessions of generalization testing. In this phase, Ss contined to receive 48 trials per session in which they were reinforced with food for making a single response to the house light. In addition, test trials with S+ and S-, in all respects exactly like the house light trials, were inserted after the 12th, 24th, 36th, and 48th house light trials. Tests with S+ and S- alternated, and which 


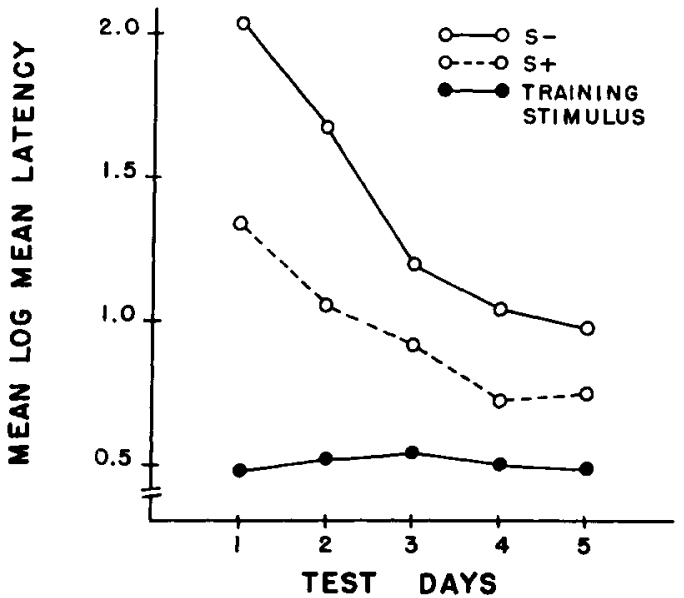

Fig. 1. Mean log mean response latency to the training stimulus and to the water-paired and unpaired generalization test stimuli for each generalization test session.

of the two test stimuli was presented first on a session was counterbalanced across Ss.

\section{Results and Discussion}

Figure 1 shows the results of generalization testing. In order to reduce the data to a more readily plottable form, and to more closely approximate the distribution requirements of analysis of variance, each S's mean latency to each of the test stimuli for each session was converted to a logarithm. Figure 1 shows the mean of these logs across Ss. The points for the training stimulus are the average across Ss of the logarithm of the average latency on the four house light trials that immediately preceded the four generalization test trials.

Large and consistent differences can be seen in the latency of responding to $S+$ and $S-$. An analysis of variance of the test trial data yielded a significant $(p<.05) \quad S+-S-$ main effect, and individual $t$ tests showed St latencies to be significantly $(p<.05)$ shorter than $S-$ latencies on all test days except the fourth.

These data clearly show that transfer from classical conditioning to instrumental responding does not depend upon the same reinforcer being used in the two stages. This finding is consistent with the results of Bower \& Kaufmann (1963). They paired one stimulus with food reinforcement and another with nonreinforcement, and then presented these two stimuli alternately during the extinction of a response previously maintained on a variable interval schedule of water reinforcement. Responding in extinction was reliably greater in the presence of the food paired stimulus than in the presence of the nonreinforcement paired stimulus.
While both the present study and the Bower and Kaufmann study show that classical $\rightarrow$ instrumental transfer of reward learning is not wholly reinforcerspecific, neither study sheds much light on the nature of the mechanism that mediates the transfer. The most salient problem is that neither study was designed to allow separation of transfer due to a facilitative effect of the reinforcement paired stimulus as opposed to an inhibitory effect of the nonreinforcement paired stimulus. It is known from other studies of classical $\rightarrow$ instrumental transfer (e.g., Trapold \& Winokur, 1967; Trapold et al, 1967) using the same reinforcer in both stages, that both types of effect do obtain. Thus, until experiments are done which allow the locus of the transfer to be isolated in the different-reinforcer case, we cannot be sure whether the present data imply cross-reinforcer generality of the facilitative effects of reinforcer-paired stimuli or the inhibitory effect of nonreinforcement paired stimuli, or both. Research designed to separate these two possible transfer components is currently in progress.

A second question left unanswered by these data is whether the amount of transfer depends upon the similarity of the reinforcers used in the classical and instrumental learning phases. All we know so far is that classical conditioning with one reinforcer will have some effect upon an instrumental response maintained by a different reinforcer. But whether the magnitude of that effect is less than or equal to that obtained when the same reinforcer is used in both phases will have strong bearing upon the kind of theoretical mechanisms that could conceivably account for classical $\rightarrow$ instrumental transfer.

\section{References}

BOWER, G., \& GRUSEC, T. Effect of prior Pavlovian discrimination training upon learning an operant discrimination. J. exp. Anal Behav., $1964,7,401-404$

BOWER, G., \& KAUFMAN, R. Transfer across drives of the discriminative effect of a Pavlovian Conditioned stimulus. J. exp. Anal. Behav., 1963, 6, 445-448.

TRAPOLD, M. A. Reversal of an operant discrimination by noncontingent discrimination reversal training. Psychon. Sci, 1966, 4, 247 248.

TRAPOLD, M. A., LAWTON, G. W., DICK, R. A., \& GROSS, D. M. Transfer of training from differential classical to differential instrumental conditioning. $J$. exp. Psychol, in press.

TRAPOLD, M. A., \& WINOKUR, S. W. Transfer from classical conditioning and extinction to acquisition, extinction, and stimulus generalization of a positively reinforced instrumental response. $J$. exp. Psychol, 1967, 73, 517.525.

Note

1. This research was supported in part by NIH Grant MH 08799 to M. A. T., and in part by grants from NICHD, NSF, and the Graduate School Research Fund of the University of Minnesota to the Center for Research in Human Learning, University of Minnesota. 